\title{
Determination of Doxorubicin Hydrochloride in Pharmaceutical Dosage Forms by a Simple Stability-Indicating Micellar Electrokinetic Capillary Chromatography Method
}

\author{
Daniele R. Nogueira-Librelotto $^{\mathrm{a}, \mathrm{b}}$, Laís E. Scheeren ${ }^{\mathrm{a}, \mathrm{b}}$, Joana R. Fernandes ${ }^{\mathrm{a}}$, Letícia B. Macedo ${ }^{\mathrm{a}, \mathrm{b}}$, Clarice M.B. Rolimª, ${ }^{\mathrm{a}, \mathrm{*}}$ \\ ${ }^{a}$ Department of Industrial Pharmacy, Universidade Federal de Santa Maria, Santa Maria, RS, Brazil \\ ${ }^{b}$ PostGraduate Program in Pharmaceutical Sciences, Universidade Federal de Santa Maria, Santa Maria, RS, Brazil \\ *Corresponding author: clarice.rolim@ufsm.br
}

\begin{abstract}
A stability-indicating micellar electrokinetic capillary chromatography (MEKC) method was developed and validated for the analysis of doxorubicin hydrochloride in injectable pharmaceutical dosage forms, using methotrexate as internal standard. A fused-silica capillary (50 $\mu \mathrm{m}$ i.d.; effective length, $40 \mathrm{~cm}$ ) and a running electrolyte solution consisting of $10 \mathrm{mM}$ borate buffer and $20 \mathrm{mM}$ anionic surfactant SDS, at $\mathrm{pH} 9.3$, were set as the best experimental conditions. Moreover, the capillary temperature was maintained at $26^{\circ} \mathrm{C}$, while the applied voltage was $+26 \mathrm{kV}$. Hydrodynamic sample injection $(6 \mathrm{~s}$ at $50 \mathrm{mbar})$ was used, and the detection was set at $260 \mathrm{~nm}$ using a photodiode array detector. The method was validated for the requirements specificity, linearity, precision, accuracy, and robustness, following the International Conference on Harmonisation (ICH) guidelines. The method linearity was proven in the range of $25-125 \mu \mathrm{g} / \mathrm{mL}(r=0.9995)$. Forced degradation studies were successfully conducted, evidencing the specificity and stability-indicating capability of the method. In addition, no interference of the excipients from the formulation was detected. The values of accuracy and precision were within the acceptable limits, and robustness studies were performed by a two-level full factorial design. The proposed method fulfilled all validation parameters and was shown to be suitable for quantitative analyses of doxorubicin, contributing, thus, to the establishment of new alternatives with advantages for the quality control of pharmaceutical formulations.
\end{abstract}

Keywords: method validation; forced degradation studies; factorial design; drug quality control; UV detection.

\section{Introduction}

Doxorubicin (DOX), a member of the anthracycline ring antibiotics, is one of the considering first-line chemotherapeutic compounds, with suitable antitumor efficacy against a wide range of solid tumors. It is a hydrophilic drug, with mechanism of action consisting in binding to DNA by intercalation, with consequent induction of a series of biochemical events that lead to apoptosis in tumor cells $(1,2)$. Cancer is one of the most common causes of death worldwide. Chemotherapy, although is considered the primary treatment for cancer among conventional modalities, displays many problems, such as low bioavailability, dose-limiting systemic toxicity and development of drug resistance (3). Even so, the administration of chemotherapies grows along with the growing number of patients with cancer. Therefore, the analytical control is extremely important to ensure the quality of the pharmaceutical formulations available for the treatment of cancer.

Concerning analytical techniques available in the literature to determine DOX, the vast majority are about liquid chromatographic (LC) methods to quantify DOX in complexes biological matrices by fluorescence $(4,5)$ or UV detector (6), as well as LC coupled to mass spectrometry (LC-MS) as a more sensitive methodology (7). With regard to the drug analysis in pharmaceutical formulations, besides the available monographs in the main pharmacopoeias worldwide describing LC-UV methods, there is also a methodology described by Sastry and Rao (8) based on complexes colorimetric reactions and spectrophotometric measurements. LC is a powerful analytical technique; however, comprises important solvent consumption, which resulted in a large volume of liquid waste contaminated with antineoplastic drugs. Likewise, the spectrophotometry might not attend the acceptable standards of sensitivity and specificity depend on the drug and/or formulation matrix.

Capillary electrophoresis (CE) has appeared as a powerful analytical technique in the pharmaceutical area, with some advantages related to the existing analytical techniques (9). As well as the LC, the CE is a closed system, which is particularly suitable in terms of safety for handling toxic samples. Moreover, it is characterized by a markedly low solvent consumption and a high versatility (10). As one of the main disadvantages, is the low sensitivity attributed to the short optical path. However, for the analysis of drugs in pharmaceutical formulations, this is not a limitation, as solutions with enough concentrations can be prepared for the analysis. Some authors have reported capillary zone electrophoresis (CZE) or micellar electrokinetic capillary chromatography (MEKC) methods to quantify DOX especially in plasma samples. GAVENDA et al. (11) used CE method to determine two anthracyclines, doxorubicin and daunorubicin (DAU), by UV detection $(190-260 \mathrm{~nm})$ using a fused-silica capillary with $50 \mu \mathrm{m}$ i.d. and $45 \mathrm{~cm}$ effective length, $100 \mathrm{mM}$ phosphate buffer $\mathrm{pH} 2.5$ added $40 \%$ (v/v) methanol as background electrolyte and $150 \mathrm{mM}$ SDS as a sweeping agent. The authors applied the method in samples of patient plasma. Moreover, CZE method was 
developed and validated in a curve range of $0.1-100$ $\mu \mathrm{g} / \mathrm{mL}$ to analyze DOX and liposomal DOX (Myocet ${ }^{\circledR}$ ) in plasma samples, using potassium phosphate buffer (12.5 $\mathrm{mM}, \mathrm{pH} 7.4)$ and laser-induced fluorescence (LIF) detection $(\lambda \mathrm{ex}=488 \mathrm{~nm}, \lambda \mathrm{em}=630 \mathrm{~nm})(12)$. Lu et al. (13) developed and validated a microchip-based CE method with LIF detection $(\lambda \mathrm{ex}=469 \mathrm{~nm}, \lambda \mathrm{em}=518 \mathrm{~nm})$ using $10 \mathrm{mM}$ sodium tetraborate buffer at $\mathrm{pH} 8.5$ with $40 \%$ acetonitrile to separate DOX and DAU. The method was linear in 1-75 $\mu \mathrm{g} / \mathrm{mL}$ range and was applied to check the recovery ratio of these drugs in human serum sample. PÉREZ-RUIZ et al. (14) developed a CE method to separate the anthracyclines DOX, DAU and idarubicin in serum samples. The method used a fused-silica capillary $75 \mu \mathrm{m}$ i.d. x $57 \mathrm{~cm}, 100 \mathrm{mM}$ borate buffer $\mathrm{pH} 9.5$ with $30 \%$ acetonitrile and appeared to be linear at $10-500 \mathrm{ng} / \mathrm{mL}$ range. MEKC was used to demonstrate the different metabolism between free and liposomal DOX (Doxil囚) treating human leukemia cells. In this case, the buffer consisted of $20 \mathrm{mM} \gamma$-cyclodextrin, 50 $\mathrm{mM}$ borate and $50 \mathrm{mM}$ SDS at $\mathrm{pH} 9.3$, with LIF detection $(\lambda \mathrm{ex}=488 \mathrm{~nm}, \lambda \mathrm{em}=635 \mathrm{~nm})(15)$. In addition, a CE method was reported to assess DOX in pharmaceutical formulations; however, this method was focused in the analysis of multiple drugs in the same experimental procedure (10). Sixteen antineoplastic drugs were determined and DOX was assessed by CZE with UV detection, using a $100 \mathrm{mM}$ phosphate buffer at $\mathrm{pH} 2.5$ containing $50 \% \mathrm{v} / \mathrm{v}$ of acetonitrile as background electrolyte, and dynamic coating of capillaries with Ceofix ${ }^{\circledR}$.

In view of these data, and considering that there is no published specific CE method for DOX determination in pharmaceutical formulations with UV detection, the objective of the present study was to develop and validate a stability-indicating MEKC method using UV-PDA detection, in accordance with ICH guidelines, as a tool to quantify DOX hydrochloride in injectable pharmaceutical dosage forms, with potential application in quality control analysis.

\section{Experimental}

\section{Chemicals and reagents}

Doxorubicin hydrochloride (DOX, state purity 98.32\%) was purchased from Zibo Ocean International Trade (Zibo, Shangdong, P.R., China) and methotrexate (MTX, state purity $100.1 \%$ ) was supplied by SM Empreendimentos Farmacêuticos Ltda (São Paulo, SP, Brazil). Different batches of Fauldoxo ${ }^{\circledR}$ (Libbs Farmacêutica Ltda., Brazil) injectable pharmaceutical solutions, containing $2 \mathrm{mg} / \mathrm{mL}$ of doxorubicin hydrochloride, were obtained within their shelf life period. Sodium dodecyl sulfate (SDS) and analytical grade boric acid were acquired from Sigma-Aldrich (São Paulo, SP, Brazil) and Merck (Darmstadt, Germany), respectively. All chemicals used were of pharmaceutical or special analytical grade. For all analyses, ultrapure water was purified using a Mega RO/UP - Mega Purity system.

\section{Apparatus}

CE experiments were performed on an Agilent 7100 Capillary Electrophoresis system (Agilent Technologies, Waldbronn, Germany) equipped with an autosampler, a 1260 Infinity photodiode array (PDA) detector, a temperature controlling system $\left(4-60^{\circ} \mathrm{C}\right)$ and power supply able to deliver up to $30 \mathrm{kV}$. A CE ChemStation software was used for instrument control, data acquisition and post run analysis of the electropherograms.

\section{Solutions}

\section{Preparation of reference substance solutions}

An exactly weighed amount of DOX was transferred to a volumetric flask and diluted to volume with ultrapure water, obtaining a reference solution with final concentration of 2 $\mathrm{mg} / \mathrm{mL}$. Methotrexate (MTX) solution, which was used as internal standard (IS), was prepared at a concentration of 1 $\mathrm{mg} / \mathrm{mL}$ using dimethylsulfoxide (DMSO). These stock solutions were kept in refrigerator, protected from light, and daily diluted to an appropriate concentration $(75 \mu \mathrm{g} / \mathrm{mL}$ for both DOX and IS) with running electrolyte solution.

\section{Preparation of sample solutions}

A proper amount of injectable pharmaceutical preparation containing $2 \mathrm{mg} / \mathrm{mL}$ of doxorubicin hydrochloride was accurately transferred to a volumetric flask and diluted to the concentration $75 \mu \mathrm{g} / \mathrm{mL}$ with running electrolyte solution. IS stock solution was also added to reach a final concentration of $75 \mu \mathrm{g} / \mathrm{mL}$. This sample solution was filtered through a $0.22 \mu \mathrm{m}$ membrane filter (Millipore) and then injected into the EC system.

\section{Preparation of running electrolyte solution}

The running electrolyte solution was prepared by dissolving separately boric acid and SDS, both equivalent to $100 \mathrm{mM}$, in ultrapure water and kept in refrigerator. Each day, the boric acid and SDS stock solutions were further diluted in order to obtain a solution with 10 and $20 \mathrm{mM}$, respectively. The $\mathrm{pH}$ was adjusted to 9.3 by adding $1 \mathrm{M}$ sodium hydroxide $(\mathrm{NaOH})$. This solution was filtered through a 0.22 $\mu \mathrm{m}$.

\section{Electrophoretic procedure}

All experiments were carried out on a fused-silica capillary with $50 \mu \mathrm{m}$ i.d. and $48.5 \mathrm{~cm}$ total length (effective length 40 $\mathrm{cm}$, Agilent), maintained at $26^{\circ} \mathrm{C}$ and with detection wavelength set at $260 \mathrm{~nm}$. The capillary was daily conditioned with $1 \mathrm{M} \mathrm{NaOH}$, ultrapure water and then the running electrolyte solution, each for 20 minutes. Between runs, the capillary was sequentially washed with $0.1 \mathrm{M}$ $\mathrm{NaOH}(180 \mathrm{~s})$, ultrapure water (180 s) and running electrolyte solution $(240 \mathrm{~s})$. With this treatment method, it was possible to achieve reproducible measurements and enhance the capillary column lifetime. The samples were 
injected by hydrodynamic method ( $6 \mathrm{~s}$ at $50 \mathrm{mbar})$ and a constant voltage of $+26 \mathrm{kV}$ was applied during the analysis. After the last run of the experiments, the capillary was rinsed with $0.1 \mathrm{M}$ sodium hydroxide, ultrapure water and finally dry cleaned.

\section{Validation of the method}

The method was validated using samples of injectable pharmaceutical formulation of doxorubicin hydrochloride with the label claim of $2 \mathrm{mg} / \mathrm{mL}$ by determinations of the following parameters: specificity, linearity, range, precision, accuracy, limit of detection (LD), limit of quantification (LQ), robustness, and system suitability test, following the International Conference on Harmonization (ICH) guidelines $(16,17)$. Samples containing $75 \mu \mathrm{g} / \mathrm{mL}$ of both DOX and IS were used as work solutions during the validation process.

\section{Forced degradation studies}

The specificity and the stability-indicating capability of the method were evaluated by forced degradation studies in order to determine the potential interferences in the quantification of DOX. Solutions containing $1000 \mu \mathrm{g} / \mathrm{mL}$ of DOX were submitted to accelerated degradation in the following conditions: acidic and alkaline hydrolysis, heat, oxidant environment with hydrogen peroxide $\left(\mathrm{H}_{2} \mathrm{O}_{2}\right)$ and UVC radiation. A sample solution prepared in $0.1 \mathrm{M}$ hydrochloric acid $(\mathrm{HCl})$ was used for the acidic hydrolysis, and a sample solution in $0.01 \mathrm{M} \mathrm{NaOH}$ for the alkaline hydrolysis evaluation. These solutions were incubated at room temperature for $24 \mathrm{~h}$ and $30 \mathrm{~min}$, respectively, and neutralized with base or acid, as necessary. To study the heat effect in the DOX degradation profile, the drug solution was diluted in water and heated in an incubator at $80{ }^{\circ} \mathrm{C}$ for $24 \mathrm{~h}$. The oxidative degradation was achieved by storing the solutions in $3 \%$ hydrogen peroxide, at room temperature for $24 \mathrm{~h}$, protected from light. Photodegradation was induced by exposing the sample in a photostability chamber to UVC radiation for $24 \mathrm{~h}$. The solutions were then diluted with the running electrolyte solution to a final DOX concentration of $75 \mu \mathrm{g} / \mathrm{mL}$. The drug content after each degradation condition was measured and the purity of both DOX and IS peaks was verified using a PDA detector (peak purity index higher than 0.999 was considered acceptable).

\section{Results and discussion}

\section{Optimization of the electrophoretic conditions}

Optimization of the electrophoretic analysis was achieved by testing different methods based on CZE or MEKC separations. Initially, a CZE method was tested, investigating some electrolyte solutions containing sodium phosphate, boric acid, phosphoric acid or biological buffers such as TRIS and HEPES at different $\mathrm{pH}$ range $(2.5-10.0)$. Indeed, it is well know that the DOX pKa is about 8.2 (18) and, thus, it can be expected that at least $50 \%$ of the drug would be ionized at this tested $\mathrm{pH}$ range. Nevertheless, no appreciable results were achieved to proceed with the method validation in none of the tested conditions. As a conclusion of these preliminary experiments, we opted to investigate the MEKC method using the surfactant SDS and borate buffer, which provided superior detector response and better analysis time in the first tests by the CZE method. Different running conditions were tested, such as buffer strength, applied voltage, $\mathrm{pH}$, as well as buffer additives. The borate buffer and SDS concentration studied ranged from 10 to $30 \mathrm{mM}$ and 10 to $50 \mathrm{mM}$, respectively, combined or not with an organic modifier. The addition of an organic modifier to the buffer has been reported to improve selectivity in MEKC (19). For this, we tested methanol or acetonitrile at small and different concentrations (5\% $20 \%$ ). Nevertheless, this option was excluded once these modifiers did not present any relevant improvements in the method optimization process. Different $\mathrm{pH}$ values for the running electrolyte solution were tested, ranging from 9.0 to 10.0 , while the applied voltage was evaluated in the range 25 to $30 \mathrm{kV}$. From these studies, it was verified the gradually increase of the current as the buffer strength and voltage increases, as well as longer run times were obtained. Therefore, the use of $10 \mathrm{mM}$ borate buffer at $\mathrm{pH} 9.3$ and SDS $20 \mathrm{mM}$ was considered the better condition for DOX analysis. It is noteworthy that similar buffers and $\mathrm{pH}$ range were used in studies mentioned elsewhere (13-15). The samples were injected hydrodynamically at 50 mbar for $6 \mathrm{~s}$ and the voltage applied was set at $+26 \mathrm{kV}$ (current about 13 $\mu \mathrm{A})$. A suitable separation performance was achieved within $8.0 \mathrm{~min}$, with a DOX migration time of $4.3 \mathrm{~min}$, showing also good peak area, intensity, symmetry and resolution. This condition showed a shorter migration time than the method developed by Pérez-Ruiz et al. (14), in which DOX was detected at about 10 minutes, but longer than $\mathrm{Lu}$ et al. (13), due to the use of a microchip by the authors. The best detection wavelength was set as $260 \mathrm{~nm}$ and, even though it is not one that correspond to the maximum absorption, was chosen due to the suitable sensitivity to both DOX and IS (Figure 1), as well as lack of interferences from the formulation excipients (Figure 2).

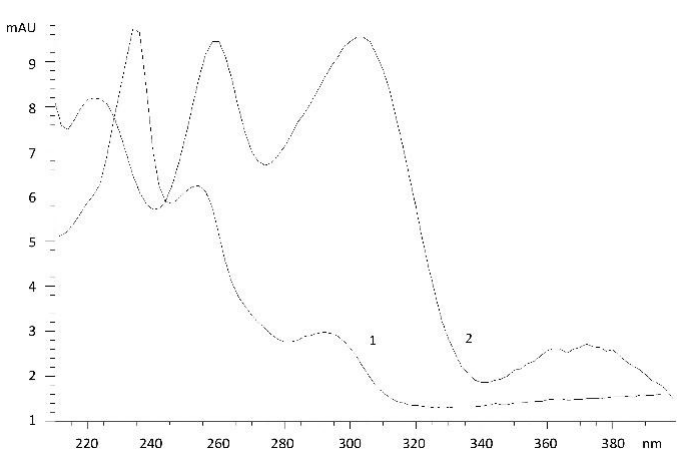

Figure 1. Absorption spectra where traces (1) and (2) correspond to DOX and MTX (IS), respectively. 


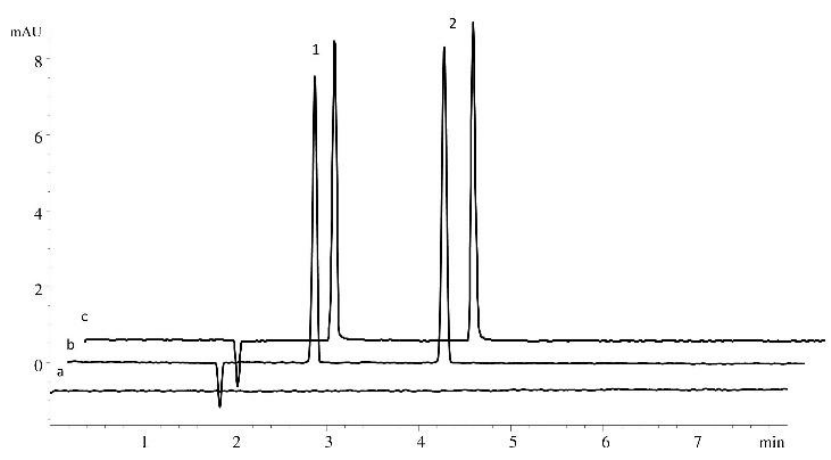

Figure 2. Representative MEKC electropherograms where trace (a) represents placebo solution, (b) DOX reference substance solution $(75 \mu \mathrm{g} / \mathrm{mL})$, and (c) DOX pharmaceutical formulation $(75 \mu \mathrm{g} / \mathrm{mL})$. Peak $1=$ internal standard $($ MTX) and peak $2=$ DOX.

The use of an IS is strongly recommended to compensate injection errors and minor fluctuations of the migration time during capillary electrophoresis analysis, thus improving the quantitative behavior. Then, some drugs, including nimesulide, furosemide and methotrexate, were tested, selecting methotrexate, another antitumor drug, which was found to be a suitable candidate, with a well resolved peak and good intensity. Figure 2 shows the typical electropherogram obtained with the optimized analytical conditions.

\section{Validation of the method}

The specificity of the method, which also determine the potential interference of the excipients of the pharmaceutical formulation, was evaluated by the analysis of placebo (an inhouse mixture of all excipients of the injectable solution without the active ingredient: sodium chloride, hydrochloric acid, sodium hydroxide and water for injection) (Figure 2). Moreover, the specificity of the method towards the drug was established through determination of peak purity of the analyte and the IS, obtained by overlay of the spectra captured at the apex, upslope and downslope using a PDA detector. Peak purity index values higher than 0.9998 demonstrated that no formulation excipients were co-eluting with DOX, thus confirming that the proposed method is specific for the analysis of DOX in injectable pharmaceutical formulations.

A stability-indicating method is as an analytical procedure that accurately quantifies the active molecule without interference from process impurities, excipients and/or degradation products. Therefore, stress testing of the drug substance can aid the identification of the potential degradation products, as well as to develop and validate the stability-indicating power of an analytical procedure (20). The forced degradation studies in neutral media followed by heat at $80^{\circ} \mathrm{C}$, as well as the photolytic condition, resulted in about 31 and $23 \%$ of drug degradation, respectively, with one eluting degradation product at the same migration time, of about $4 \mathrm{~min}$ (Figures $3 \mathrm{a}$ and $\mathrm{b}$ ). Under the alkaline hydrolysis, DOX content exhibited a significant decrease after just $30 \mathrm{~min}$ of exposition, and one additional resolved peak was detected at $3 \mathrm{~min}$ (Figure 3c). In contrast, the acidic condition, only $12 \%$ of DOX degradation was observed after $24 \mathrm{~h}$ and no additional peak was detected in the electropherogram (Figure 3d), indicating that the degradation products did not migrate in the MEKC method or may have been degraded to nonchromophoric products. Finally, the oxidative condition also exhibited decrease of the area, with three additional peaks detected at about 3,4 and $7.8 \mathrm{~min}$ (Figure 3e). Table 1 shows the percentage of drug degradation for each stress condition. Peak purity analysis were also performed in all tested stress conditions, and acceptable values (> 0.9998) were obtained for both DOX and IS peaks.

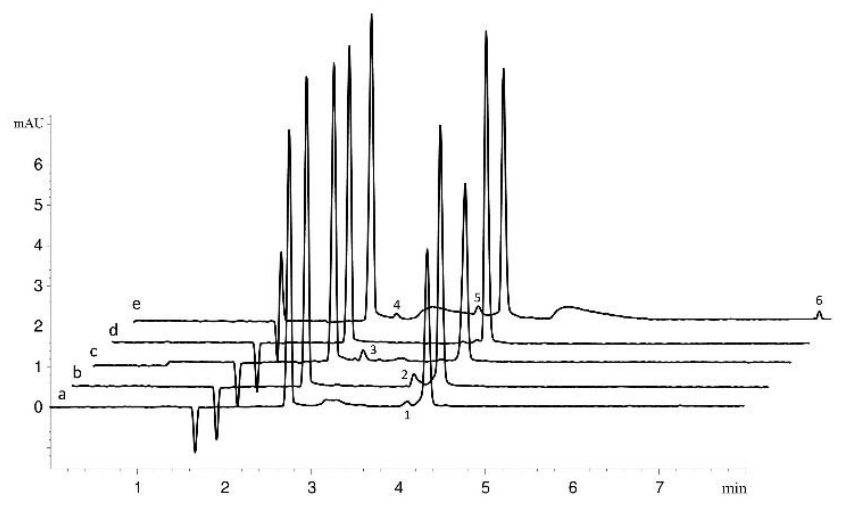

Figure 3. Typical MEKC electropherograms of DOX solution (75 $\mu \mathrm{g} / \mathrm{mL}$ ) after degradation under (a) neutral hydrolysis followed by heat at $80^{\circ} \mathrm{C}$, (b) photolytic condition, (c) alkaline hydrolysis, (d) acidic hydrolysis, and (e) oxidative condition. Peaks 1 to 6 correspond to DOX degraded forms.

Table 1. Amount of DOX degraded in each stress condition

\begin{tabular}{|c|c|c|}
\hline Condition & $\begin{array}{l}\text { DOX degradation }^{\mathrm{a}} \\
(\%) \pm \mathrm{SD}^{\mathrm{b}}\end{array}$ & $\operatorname{RSD}^{\mathrm{c}}(\%)$ \\
\hline $0.01 \mathrm{M} \mathrm{NaOH}$ for $0.5 \mathrm{~h}$ & $37.05 \pm 2.28$ & 5.12 \\
\hline $0.1 \mathrm{M} \mathrm{HCl}$ for $24 \mathrm{~h}$ & $12.36 \pm 1.96$ & 3.16 \\
\hline UVC radiation for $24 \mathrm{~h}$ & $23.17 \pm 4.77$ & 6.78 \\
\hline $\begin{array}{l}\text { Oxidation with } \mathrm{H}_{2} \mathrm{O}_{2} \text { for } \\
\qquad 24 \mathrm{~h}\end{array}$ & $27.07 \pm 3.28$ & 6.37 \\
\hline $\begin{array}{c}\text { Neutral hydrolysis at } 80^{\circ} \mathrm{C} \\
\text { for } 24 \mathrm{~h}\end{array}$ & $31.32 \pm 8.79$ & 6.10 \\
\hline
\end{tabular}

Linearity was determined by constructing three independent analytical curves in the range of $25-125 \mu \mathrm{g} / \mathrm{mL}$ prepared in running electrolyte solution. Three replicate injections of each reference solution spiked with MTX $(75 \mu \mathrm{g} / \mathrm{mL})$ as IS, were made to verify the repeatability of the detector response. The peak area ratio of DOX to the IS, against the respective reference concentrations, was used for plotting the graph, and the linearity evaluated by the least square regression analysis. The MEKC method was linear ( $\mathrm{r}=$ $0.9995, \mathrm{y}=0.0142 \mathrm{x}+0.0179$, where $x$ is concentration of DOX expressed in $\mu \mathrm{g} / \mathrm{mL}$ and $y$ is the peak area ratio of DOX to IS). 
The LD and LQ were calculated by using the mean of the slope $(S)$ and the standard deviation of the intercept $(\sigma)$ of three independent analytical curves, determined by a linear regression model, as defined by ICH (16). The theoretical values obtained were 4.60 and $15.32 \mu \mathrm{g} / \mathrm{mL}$, for $\mathrm{LD}$ and $\mathrm{LQ}$, respectively. Even though these parameters are not a requirement for drug assay, they are certainly important to evidence the method sensitivity.

The precision of the method was determined by repeatability (intra-day precision) and intermediate precision (inter-day and between-analysts precision), and the experimental results were expressed as relative standard deviation (RSD). Repeatability was examined by calculating the RSD of assay results $(\%)$ of six independent sample preparations at 75 $\mu \mathrm{g} / \mathrm{mL}$. The intermediate precision was assessed by analyzing three samples of the pharmaceutical formulation on two different days (inter-days) and by a second analyst (between-analysts). The precision results showed all RSD values lower than $2.0 \%$, as shown in Table 2, demonstrating that the method variability was within the acceptable range.

Table 2. Repeatability ${ }^{\ddagger}$, intermediate precision ${ }^{\S}$ and accuracy data of MEKC method for doxorubicin analysis in samples of injectable pharmaceutical formulations.

\begin{tabular}{|c|c|c|}
\hline \multicolumn{3}{|c|}{ Precision } \\
\hline & Assay $\pm \mathrm{SD}^{\mathrm{a}}$ & $\operatorname{RSD}^{\mathrm{b}}(\%)$ \\
\hline Intra-day $(n=6)$ & $99.76 \pm 1.279$ & 1.28 \\
\hline \multicolumn{3}{|l|}{ Inter-day } \\
\hline Day $1(n=3)$ & $101.47 \pm 0.783$ & 0.77 \\
\hline Day $2(n=3)$ & $102.47 \pm 1.576$ & 1.54 \\
\hline Mean $(n=6)$ & $101.97 \pm 0.708$ & 0.69 \\
\hline \multicolumn{3}{|l|}{ Between-analysts } \\
\hline Analyst $1(\mathrm{n}=3)$ & $102.47 \pm 1.576$ & 1.54 \\
\hline Analyst $2(\mathrm{n}=3)$ & $104.11 \pm 0.679$ & 0.65 \\
\hline Mean $(n=6)$ & $103.29 \pm 1.158$ & 1.12 \\
\hline \multicolumn{3}{|c|}{ Accuracy } \\
\hline & Recovery \pm SD & $\operatorname{RSD}(\%)$ \\
\hline $60 \mu \mathrm{g} / \mathrm{mL}$ & $101.79 \pm 0.002$ & 0.23 \\
\hline $75 \mu \mathrm{g} / \mathrm{mL}$ & $101.27 \pm 0.002$ & 0.17 \\
\hline $90 \mu \mathrm{g} / \mathrm{mL}$ & $99.22 \pm 0.012$ & 0.77 \\
\hline Mean & $100.76 \pm 1.36$ & 1.35 \\
\hline
\end{tabular}

intra-day

$\S$ inter-day and between-analysts

${ }^{\text {a }} \mathrm{SD}$ : Standard deviation

${ }^{\mathrm{b}} \mathrm{RSD}$ : Relative standard deviation

The accuracy was evaluated by the recovery of known amounts of reference solution, added to a sample solution, to obtain solutions at concentrations of 60,75 , and $90 \mu \mathrm{g} / \mathrm{mL}$, equivalent to 80,100 , and $120 \%$, of the analytical concentration $(75 \mu \mathrm{g} / \mathrm{mL})$, respectively. The accuracy was calculated at each concentration level from differences between the responses obtained for spiked and unspiked solutions. The absolute recovery values obtained for DOX are shown in Table 2, with a satisfactory mean percentage of $100.76 \%$, evidencing, thus, the accuracy of the method within the desired range.

The robustness was assessed by evaluating the capability of the method to remain unaffected by small, but deliberate, modifications in some of the methods settings. A two-level full factorial design (number of analyses equal to $2^{\mathrm{k}}+\mathrm{n}$, where $k$ is the number of factors and $n$ is the number of central points) was performed using Minitab v.17 software (Minitab Inc., State College, PA, USA). Here, a $2^{3}$ full factorial was applied, by analyzing samples after small changes in one, two or all of the following factors: borate buffer concentration in the electrolyte solution (8 and 12 $\mathrm{mM})$, capillary temperature $\left(24\right.$ and $28{ }^{\circ} \mathrm{C}$ ) and voltage applied (24 and $28 \mathrm{kV})$. Therefore, second- and third-order interactions were evaluated (21). The analysis of the DOX reference and sample solutions containing $75 \mu \mathrm{g} / \mathrm{mL}$ were carried out in each combination, in a randomized order. The results were expressed as a Pareto chart (Figure 4), which shows that the single factors or their combinations did not significantly influence the analytical performance, since the bars did not cross the vertical line (critical $t$-value for $\alpha=$ 0.05). Moreover, there were non-significant changes of the migration time and peak area ratios, as well as of the electropherogram pattern, in comparison to the optimized conditions, indicating that the proposed method is robust under the conditions tested.

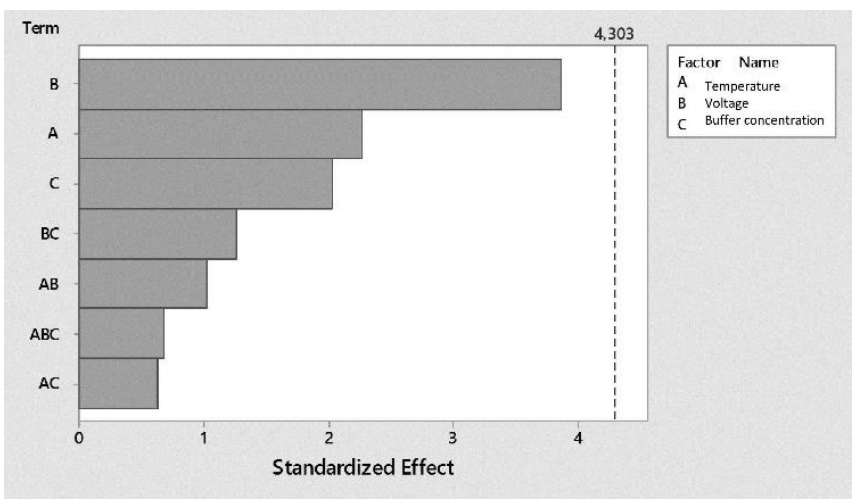

Figure 4. Pareto chart of the robustness evaluation of the MEKC method. The horizontal columns represent the main factors and their combinations. The vertical line indicates the critical $t$-value.

A system suitability test was also carried out to evaluate the adequate performance and reproducibility of the system for the analysis to be executed, using six replicates injections of a reference substance solution containing $75 \mu \mathrm{g} / \mathrm{mL}$ of DOX and IS. The RSD values calculated for the migration time and peak area were 1.74 and $1.82 \%$, respectively. The number of theoretical plates and peak symmetry were about 27597 and 1.03, with RSD of 1.65 and $1.05 \%$, respectively. The resolution between DOX and IS peaks was 16.48 , with RSD of $1.35 \%$. Altogether, the experimental data showed that the parameters tested were within the acceptable range (RSD $<2.0 \%$ ), indicating that the method is suitable for the analysis intended. 


\section{Method application}

The validated MEKC method was applied for the determination of DOX in four different batches of an injectable pharmaceutical formulation. All batches showed mean content value in the range of 101.0 to $103.0 \%$, with RSD lower than $1.0 \%$. Therefore, the proposed method can be useful for the determination of DOX in injectable formulations, without prior separation of the excipients, with advantages of small sample volumes, short analysis time and no consumption of organic solvents, with the exception of a small amount of DMSO used to prepare the stock solution of the internal standard MTX.

\section{Conclusion}

The results of the validation studies show that the MEKC method is specific and stability-indicating. It possesses appropriate linearity, together with precision and accuracy within the acceptable limits. A full factorial design was successfully applied to assess robustness with many advantages over the traditional one-variable-at-time approach. The proposed method was also effectively applied for the quantitative analysis of doxorubicin in injectable pharmaceutical formulations, representing, thus, a simple and environment-friendly alternative for the quality control laboratory.

\section{Conflict of interest statement}

The authors state that they have no conflict of interest.

\section{Acknowledgements}

This research was supported by Grants 447548/2014-0 e 401069/2014-1 from Conselho Nacional de Desenvolvimento Científico e Tecnológico (CNPq - Brazil). Daniele R. Nogueira-Librelotto wish to thank CNPq for the postdoctoral fellowship.

\section{References}

1. Shalviri, A.; Raval, G.; Prasad, P.; Chan, C.; Liu, Q.; Heerklotz, H.; Rauth, A.M.; Wu, X.Y. pH-Dependent doxorubicin release from terpolymer of starch, polymethacrylic acid and polysorbate 80 nanoparticles for overcoming multi-drug resistance in human breast cancer cells. Eur. J. Pharm. Biopharm. 2012; 82:587-597.

2. Xiong, X.-B.; Ma, Z.; Lai, R.; Lavasanifar, A. The therapeutic response to multifunctional polymeric nanoconjugates in the targeted cellular and subcellular delivery of doxorubicin. Biomaterials. 2010; 31:757-768.

3. Chen, Y.; Zhang, W.; Huang, Y.; Gao, F.; Sha, X.; Fang, $\mathrm{X}$. Pluronic-based functional polymeric mixed micelles for co-delivery of doxorubicin and paclitaxel to multidrug resistant tumor. Int J Pharm. 2015; 488:44-58.

4. Gilbert, C.M.; Mcgearya, R.P.; Filippich, L.J.; Norris, R.L.G.; Charles, B.G. Simultaneous liquid chromatographic determination of doxorubicin and its major metabolite doxorubicinol in parrot plasma. J. Chromatogr. B. 2005; 826:273-276.

5. Urva, S.R.; Shin, B.S.; Yang, V.C.; Balthasar, J.P. Sensitive high performance liquid chromatographic assay for assessment of doxorubicin pharmacokinetics in mouse plasma and tissues. J. Chromatogr. B. 2009; 887:837-841.

6. Ahmad, M.; Usman, M.; Madni, A.; Zubair, M.; QamarUz-Zaman, M.; Qureshi, M.S.; Munir, A.; Ahmad, M.; Mahmood, A. A fast and simple HPLC-UV method for simultaneous determination of three anti-cancer agents in plasma of breast cancer patients and its application to clinical pharmacokinetics. African J. Pharm. Pharmacol. 2011; 5:915-922.

7. Ma, W.; Wang, J.; Guo, Q.; Tu, P. Simultaneous determination of doxorubicin and curcumin in rat plasma by LC-MS/MS and its application to pharmacokinetic study. J. Pharm. Biomed. Anal. 2015; 111:215-221.

8. Sastry, C.S.; Rao, J.S.L. Determination of doxorubicin hydrochloride by visible spectrophotometry. Talanta. 1996; 43:1827-1835.

9. Kapnissi-Christodoulou, C.P. Method Development by Use of Capillary Electrophoresis and Applications in Pharmaceutical, Biological and Natural Samples In: Electrophoresis, Kiumars Ghowsi, Ed., IntechOpen, 2012. Doi: $10.5772 / 45834$.

10. Guichard, N.; Ogereau, M.; Falaschi, L.; Rudaz, S.; Schappler, J.; Bonnabry, P.; Fleury-Souverain, S. Determination of 16 antineoplastic drugs by capillary electrophoresis with UV detection: Applications in quality control. Electrophoresis. 2018; 39:2512-2520.

11. Gavenda, A.; Sevcik, J.; Psotova, J.; Bednar, P.; Bartak, P.; Adamovsky, P.; Simanek, V. Determination of anthracycline antibiotics doxorubicin and daunorubicin by capillary electrophoresis with UV absorption detection. Electrophoresis. 2001; 22:2782-2785.

12. Kim, S.E.; Wainer, I.W. Simultaneous analysis of liposomal doxorubicin and doxorubicin using capillary electrophoresis and laser induced fluorescence. J. Pharm. Biomed. Anal. 2010; 52:372-376.

13. Lu, H.; Yuan, G.; He, Q.; Chen, H. Rapid analysis of anthracycline antibiotics doxorubicin and daunorubicin by 
microchip capillary electrophoresis. Microchem. J. 2009; 92:170-173.

14. Pérez-Ruiz, T.; Martínez-Lozano, C.; Sanz, A.; Bravo, E. Simultaneous determination of doxorubicin, daunorubicin, and idarubicin by capillary electrophoresis with laser-induced fluorescence detection. Electrophoresis. 2001; 22:134-138.

15. Eder, A. R.; Arriaga, E. A. Micellar electrokinetic capillary chromatography reveals differences in intracellular metabolism between liposomal and free doxorubicin treatment of human leukemia cells. J. Chromatogr. B. 2005; 829:115-122.

16. International Conference on Harmonization (ICH) of Technical Requirements for the Registration of Pharmaceutical for Human Use. Validation of Analytical Procedures: Text and Methodology Q2(R1), Geneva, Switzerland, November, 2005, 17p.

17. International Conference on Harmonization ( $\mathrm{ICH})$ of Technical Requirements for the Registration of Pharmaceutical for Human Use. Stability Testing of New Drugs Substance and Products Q1A(R2), Geneva, Switzerland, February, 2003, 18p.

18. Tavano, L.; Aiello, R.; Ioele, G.; Picci, N.; Muzzalupo, R. Niosomes from glucuronic acid-based surfactant as new carriers for cancer therapy: preparation, characterization and biological properties. Colloids Surf B Biointerfaces. 2014; 118:7-13.

19. Bourdon, F.; Lecoeur, M.; Duhaut, M.; Odou, P.; Vaccher, C.; Foulon, C. A validated micellar electrokinetic chromatography method for the quantitation of dexamethasone, ondansetron and aprepitant, antiemetic drugs, in organogel. J. Pharm. Biomed. Anal. 2013; 86:4048 .

20. Chilukuri, M.; Narayanareddy, P.; Hussianreddy, K. Stability-indicating HPLC method for determination of fosamprenavir calcium. J. Chromatogr. Sci. 2014; 52:781787.

21. Teófilo, R.F.; Ferreira, M.M.C. Quimiometria II: planilhas eletrônicas para cálculos de planejamentos experimentais, um tutorial. Quim. Nova. 2006; 29:338-350. 